\title{
Explicit expression for symmetric identities of $w$-Catalan-Daehee polynomials
}

\author{
Taekyun Kim ${ }^{1}$, Seog-Hoon Rim ${ }^{2}$, \\ Dmitry V. Dolgy ${ }^{3}$ and Sung-Soo Pyo ${ }^{4}$ \\ ${ }^{1}$ Department of Mathematics, Tianjin Polytechnic University \\ Tianjin 300387, China, and \\ Department of Mathematics, Kwangwoon University \\ Seoul, Republic of Korea \\ e-mail: tkkimekw.ac.kr \\ ${ }^{2}$ Department of Mathematics Education, Kyungpook National University \\ Daegu, Republic of Korea \\ e-mail: shrimeknu.ac.kr \\ ${ }^{3}$ Institute of Natural Sciences, Far Eastern Federal University \\ Vladivostok, 690950, Russia \\ e-mail: d_dol@mail.ru \\ ${ }^{4}$ Department of Mathematics Education, Silla University \\ Busan, Republic of Korea \\ e-mail: ssoopyo@gmail.com
}

Received: 19 September 2018

Accepted: 26 October 2018

\begin{abstract}
Recently, Catalan-Daehee numbers are studied by several authors. In this paper, we consider the $w$-Catalan-Daehee polynomials and investigate some properties for those polynomials. In addition, we give explicit expression for the symmetric identities of the $w$-Catalan-Daehee polynomials which are derived from $p$-adic invariant integral on $\mathbb{Z}_{p}$.
\end{abstract}

Keywords: Catalan numbers, Daehee numbers, $w$-Catalan-Daehee numbers.

2010 Mathematics Subject Classification: 11B83, 11 S80. 


\section{Introduction}

In combinatorial mathematics, the Catalan numbers form a sequence of natural numbers that occur in various counting problems, often involving recursively defined objects. The $n$-th Catalan numbers are defined in the terms of binomial coefficients which are given by

$$
C_{n}=\frac{1}{n+1}\left(\begin{array}{c}
2 n \\
n
\end{array}\right)=\frac{(2 n) !}{(n+1) ! n !}=\prod_{k=2}^{n} \frac{n+k}{k}(n \geq 0) .
$$

The generating function of Catalan number is given by

$$
\frac{1-\sqrt{1-4 t}}{2 t}=\frac{2}{1+\sqrt{1-4 t}}=\sum_{n=0}^{\infty} C_{n} t^{n},(\text { see }[12,15,25]) .
$$

In addition, the Catalan polynomials are also defined by the generating function to be

$$
\frac{2}{1+\sqrt{1-4 t}}(1-4 t)^{\frac{x}{2}}=\sum_{n=0}^{\infty} C_{n}(x) \frac{t^{n}}{n !}(\text { see }[10,14]) .
$$

It is easy to show that the expression of $\sqrt{1+t}$ is given by

$$
\sqrt{1+t}=\sum_{k=0}^{\infty}(-1)^{k-1}\left(\begin{array}{c}
2 k \\
k
\end{array}\right) \frac{1}{4^{k}}\left(\frac{1}{2 k-1}\right) t^{k} .
$$

Replacing $t$ by $-4 t$ in (2), we have the generating function of Catalan numbers (1),

$$
\begin{aligned}
\sqrt{1-4 t} & =1-2 \sum_{m=0}^{\infty}\left(\begin{array}{c}
2 m \\
m
\end{array}\right) \frac{1}{m+1} t^{m+1} \\
& =1-2 \sum_{m=0}^{\infty} C_{m} t^{m+1} .
\end{aligned}
$$

It is well known that the Daehee numbers, denoted by $D_{n}$, are defined by the generating function

$$
\frac{\log (1+t)}{t}=\sum_{n=0}^{\infty} D_{n} \frac{t^{n}}{n !}
$$

Even though the Daehee numbers are easily calculated as $D_{n}=(-1)^{n} \frac{n !}{n+1}$, they play important roles in connecting relationships between special numbers (see $[3,5,7,9,18,20-25]$ ).

The Catalan-Daehee numbers are defined by assigning $\sqrt{1-4 t}-1$ instead of $t$ in the definition of Daehee numbers (3), as follows:

$$
\frac{\frac{1}{2} \log (1-4 t)}{\sqrt{1-4 t}-1}=\sum_{n=0}^{\infty} d_{n} t^{n},(\text { see }[3,14]) .
$$


From (4), we note that

$$
\begin{aligned}
\frac{\frac{1}{2} \log (1-4 t)}{\sqrt{1-4 t}-1} & =\frac{1}{2} \sum_{l=0}^{\infty} \frac{4^{l}}{l+1} t^{l}\left(2-2 \sum_{m=0}^{\infty} C_{m} t^{m+1}\right) \\
& =\sum_{l=0}^{\infty} \frac{4^{l}}{l+1} t^{l}\left(1-\sum_{m=0}^{\infty} C_{m} t^{m+1}\right) \\
& =\sum_{n=0}^{\infty} \frac{4^{n}}{n+1} t^{n}-\sum_{l=0}^{\infty} \frac{4^{l}}{l+1} t^{l} \sum_{m=0}^{\infty} C_{m} t^{m+1} \\
& =1+\sum_{n=1}^{\infty}\left(\frac{4^{n}}{n+1}-\sum_{m=0}^{n-1} \frac{4^{n-m-1}}{n-m} C_{m}\right) t^{n}
\end{aligned}
$$

From (4) and (5), we can derive the following equation (6)

$$
\begin{aligned}
d_{n} & = \begin{cases}1, & \text { if } n=0 \\
\frac{4^{n}}{n+1}-\sum_{m=0}^{n-1} \frac{4^{n-m-1}}{n-m} C_{m}, & \text { if } n \geq 1,\end{cases} \\
& =-\sum_{m=0}^{n} \frac{4^{n-m}}{n-m+1} C_{m-1}^{*}, \text { for all } n \geq 0,
\end{aligned}
$$

where

$$
C_{m-1}^{*}= \begin{cases}-1 & \text { if } m=0 \\ C_{m-1} & \text { if } m \geq 1\end{cases}
$$

From the generating function (1), a kind of generalization of the Catalan numbers, the so called $w$-Catalan numbers are introduced in [10], as follows:

$$
\frac{2}{1+\sqrt{(1-4 t)^{w}}}(1-4 t)^{\frac{w}{2} x}=\sum_{n=0}^{\infty} C_{n, w}(x) t^{n} .
$$

Recently, a group of mathematicians studied the symmetric identities of special polynomials which are derived from the $p$-adic invariant integral on $\mathbb{Z}_{p}$ (see $[1,2,4,6,8,11,17,20]$ ).

In this paper, we define $w$-Catalan-Daehee polynomials and numbers. We give some identities for $w$-Catalan-Daehee polynomials and numbers. In addition, we give some new explicit expression for $w$-Catalan-Daehee numbers which are derived from $p$-adic integrals on $\mathbb{Z}_{p}$.

\section{The $w$-Catalan-Daehee polynomials}

For $w \in \mathbb{N}$, we define the $w$-Catalan-Daehee polynomials, $d_{n, w}(x)$, using the generating function, as follows:

$$
\frac{\frac{1}{2} \log (1-4 t)}{\sqrt{(1-4 t)^{w}}-1}(1-4 t)^{\frac{w x}{2}}=\sum_{n=0}^{\infty} d_{n, w}(x) t^{n} .
$$

Note that $\lim _{w \rightarrow 1} d_{n, w}(x)=d_{n}(x),(n \geq 0)$, and we call $d_{n, w}=d_{n, w}(0) w$-Catalan-Daehee numbers. 
From the definition of $w$-Cataln-Daehee numbers and Daehee numbers,

$$
\begin{aligned}
\frac{\frac{1}{2} \log (1-4 t)}{\sqrt{(1-4 t)^{w}}-1} & =\frac{\frac{1}{w} \log \left((1-4 t)^{\frac{w}{2}}-1+1\right)}{(1-4 t)^{\frac{w}{2}}-1} \\
& =\frac{1}{w} \sum_{l=0}^{\infty} D_{n} \frac{\left((1-4 t)^{\frac{w}{2}}-1\right)^{l}}{l !} \\
& =\frac{1}{w} \sum_{l=0}^{\infty} \frac{D_{n}}{l !} \sum_{k=0}^{l}\left(\begin{array}{l}
l \\
k
\end{array}\right)(1-4 t)^{\frac{w}{2} k}(-1)^{l-k} \\
& =\frac{1}{w} \sum_{l=0}^{\infty} \frac{D_{n}}{l !} \sum_{k=0}^{l} \sum_{i=0}^{\infty}\left(\begin{array}{l}
l \\
k
\end{array}\right)\left(\begin{array}{c}
\frac{w}{2} k \\
i
\end{array}\right)(-4 t)^{i}(-1)^{l-k} \\
& =\sum_{n=0}^{\infty}\left(\frac{1}{w} \sum_{l=0}^{\infty} \sum_{k=0}^{l}(-1)^{n+l-k} \frac{D_{n}}{l !}\left(\begin{array}{l}
l \\
k
\end{array}\right)\left(\begin{array}{c}
\frac{w}{2} k \\
n
\end{array}\right) 4^{n}\right) t^{n}
\end{aligned}
$$

From the equation (8) and (9), we have a relation between the $w$-Catalan-Daehee and Daehee numbers.

Proposition 1. For any $w, n \in \mathbb{N}$,

$$
d_{n, w}=\frac{1}{w} \sum_{l=0}^{\infty} \sum_{k=0}^{l}(-1)^{n+l-k} \frac{D_{n}}{l !}\left(\begin{array}{l}
l \\
k
\end{array}\right)\left(\begin{array}{c}
\frac{w}{2} k \\
n
\end{array}\right) 4^{n} .
$$

The following can be obtained from the definition of the $w$-Catalan-Daehee polynomials.

$$
\begin{aligned}
\frac{\frac{1}{2} \log (1-4 t)}{\sqrt{(1-4 t)^{w}}-1}(1-4 t)^{\frac{w x}{2}} & =\left(\frac{1}{2} \sum_{l=1}^{\infty} \frac{4^{l}}{l} t^{l}\right)\left(\sum_{k=0}^{\infty}(1-4 t)^{\frac{w}{2} k}\right)(1-4 t)^{\frac{w x}{2}} \\
& =\left(\frac{1}{2} \sum_{l=1}^{\infty} \frac{4^{l}}{l} t^{l}\right)\left(\sum_{k=0}^{\infty}(1-4 t)^{\frac{w}{2}(k+x)}\right) \\
& =\left(\frac{1}{2} \sum_{l=1}^{\infty} \frac{4^{l}}{l} t^{l}\right)\left(\sum_{k=0}^{\infty} \sum_{i=0}^{\infty}\left(\begin{array}{c}
\frac{w}{2}(k+x) \\
i
\end{array}\right)(-4)^{i} t^{i}\right) \\
& =\sum_{n=0}^{\infty}\left(\sum_{l=1}^{n} \sum_{k=0}^{\infty} \frac{1}{2} \frac{4^{l}}{l}\left(\begin{array}{c}
\frac{w}{2}(k+x) \\
n-l
\end{array}\right)(-4)^{n-l}\right) t^{n}
\end{aligned}
$$

The equation (10) gives us an explicit formula for the $w$-Catalan-Daehee polynomials.

Proposition 2. For any $w, n \in \mathbb{N}$,

$$
d_{n, w}(x)=\sum_{l=1}^{n} \sum_{k=0}^{\infty} \frac{1}{2} \frac{4^{l}}{l}\left(\begin{array}{c}
\frac{w}{2}(k+x) \\
n-l
\end{array}\right)(-4)^{n-l} .
$$

It is natural to look for for a relationship between Daehee numbers and $w$-Catalan-Daehee numbers. For this, substituting $\frac{1-\left(1+t_{0}\right)^{\frac{2}{w}}}{4}$ instead of $t$ in the definition of $w$-Catalan-Daehee numbers (8), the left side becomes 


$$
\frac{\frac{1}{2} \log \left(1-4\left(\frac{1-(1+t)^{\frac{2}{w}}}{4}\right)\right)}{\sqrt{\left(1-4\left(\frac{1-(1+t) \frac{2}{w}}{4}\right)\right)^{w}-1}}=\frac{\frac{1}{w} \log (1+t)}{t}=\sum_{n=0}^{\infty} \frac{D_{n}}{w} \frac{t^{n}}{n !}
$$

and the right side becomes

$$
\begin{aligned}
\sum_{l=0}^{\infty} d_{l, w}\left(\frac{1-\left(1+t_{0}\right)^{\frac{2}{w}}}{4}\right)^{l} & =\sum_{l=0}^{\infty} \frac{d_{l, w}}{4^{l}} \sum_{k=0}^{l}(-1)^{k}(1+t)^{\frac{2}{w} k} \\
& =\sum_{l=0}^{\infty} \frac{d_{l, w}}{4^{l}} \sum_{k=0}^{l}(-1)^{k} \sum_{i=0}^{\infty}\left(\begin{array}{c}
\frac{2}{w} k \\
i
\end{array}\right) t^{i} \\
& =\sum_{n=0}^{\infty}\left(\sum_{l=0}^{\infty} \sum_{k=0}^{l} \frac{d_{l, w}}{4^{l}}(-1)^{k}\left(\begin{array}{c}
\frac{2}{w} k \\
n
\end{array}\right)\right) t^{n}
\end{aligned}
$$

From (11) and (12), we get the following

Proposition 3. For any $w, n \in \mathbb{N}$,

$$
D_{n}=w \sum_{l=0}^{\infty} \sum_{k=0}^{l} \frac{d_{l, w}}{4^{l}}(-1)^{k}\left(\begin{array}{c}
\frac{2}{w} k \\
n
\end{array}\right) .
$$

To observe relations between Catalan numbers and $w$-Catalan-Daehee numbers, substitute $\frac{1-(2+\sqrt{1-4 t})^{\frac{2}{w}}}{4}$ for $t$ in the definition of $w$-Catalan-Daehee numbers.

$$
\begin{aligned}
\frac{\frac{1}{2} \log \left(1-4\left(\frac{1-(2+\sqrt{1-4 t})^{\frac{2}{w}}}{4}\right)\right)}{\sqrt{\left(1-4\left(\frac{1-(2+\sqrt{1-4 t})^{\frac{2}{w}}}{4}\right)\right)^{w}}-1} \\
=\frac{\frac{1}{w} \log (2+\sqrt{1-4 t})}{1+\sqrt{1-4 t}} \\
=-\frac{1}{2 w} \sum_{k=1}^{\infty} \frac{(-1)^{k}}{k}(1+\sqrt{1-4 t})^{k} \sum_{m=0}^{\infty} C_{m} t^{m} \\
=-\frac{1}{2 w} \sum_{k=1}^{\infty} \frac{(-1)^{k}}{k} \sum_{l=0}^{k}\left(\begin{array}{l}
k \\
l
\end{array}\right)(1-4 t)^{\frac{l}{2}} \sum_{m=0}^{\infty} C_{m} t^{m} \\
=-\frac{1}{2 w} \sum_{k=1}^{\infty} \frac{(-1)^{k}}{k} \sum_{l=0}^{k}\left(\begin{array}{l}
k \\
l
\end{array}\right) \sum_{i=0}^{\infty}\left(\begin{array}{c}
\frac{l}{2} \\
i
\end{array}\right)(-4 t)^{i} \sum_{m=0}^{\infty} C_{m} t^{m} \\
=-\frac{1}{2 w} \sum_{k=1}^{\infty} \frac{(-1)^{k}}{k} \sum_{l=0}^{k}\left(\begin{array}{l}
k \\
l
\end{array}\right) \sum_{j=0}^{\infty} \sum_{i=0}^{j}\left(\begin{array}{l}
\frac{l}{2} \\
i
\end{array}\right)(-4)^{i} C_{j-i} t^{j} \\
=\sum_{n=0}^{\infty}\left(\sum_{k=1}^{\infty} \sum_{l=0}^{k} \sum_{i=0}^{n} \frac{1}{2 w} \frac{(-1)^{i+k+1}}{k}\left(\begin{array}{l}
k \\
l
\end{array}\right)\left(\begin{array}{l}
\frac{l}{2} \\
i
\end{array}\right)(-4)^{i} C_{n-i}\right) t^{n} .
\end{aligned}
$$


And the right side becomes

$$
\begin{aligned}
\sum_{k=0}^{\infty} d_{n, w} & \left(\frac{1-(2+\sqrt{1-4 t})^{\frac{2}{w}}}{4}\right)^{k} \\
= & \sum_{k=0}^{\infty} \frac{d_{n, w}}{4^{k}} \sum_{l=0}^{k}(-1)^{l}(1+\sqrt{1-4 t})^{\frac{2}{w}} l \\
= & \sum_{k=0}^{\infty} \frac{d_{n, w}}{4^{k}} \sum_{l=0}^{k}(-1)^{l} \sum_{i=0}^{\infty}\left(\begin{array}{c}
\frac{2}{w} l \\
i
\end{array}\right)(1-4 t)^{\frac{i}{2}} \\
= & \sum_{k=0}^{\infty} \frac{d_{k, w}}{4^{k}} \sum_{l=0}^{k}(-1)^{l} \sum_{i=0}^{\infty}\left(\begin{array}{c}
\frac{2}{w} l \\
i
\end{array}\right) \sum_{j=0}^{\infty}\left(\begin{array}{c}
\frac{i}{2} \\
j
\end{array}\right)(-4 t)^{j} \\
= & \sum_{n=0}^{\infty}\left(\sum_{k=0}^{\infty} \frac{d_{k, w}}{4^{k}} \sum_{l=0}^{k} \sum_{i=0}^{\infty}(-1)^{n+l} 4^{n}\left(\begin{array}{c}
\frac{2}{w} l \\
i
\end{array}\right)\left(\begin{array}{c}
\frac{i}{2} \\
j
\end{array}\right)\right) t^{n}
\end{aligned}
$$

From the equation (13) and (14), we have the following identity between the Catalan and $w$-Catalan-Daehee numbers.

Proposition 4. For any $w, n \in \mathbb{N}$,

$$
\sum_{k=1}^{\infty} \sum_{l=0}^{k} \sum_{i=0}^{n} \frac{1}{2 w} \frac{(-1)^{i+k+1}}{k}\left(\begin{array}{c}
k \\
l
\end{array}\right)\left(\begin{array}{c}
\frac{l}{2} \\
i
\end{array}\right)(-4)^{i} C_{n-i}=\sum_{k=0}^{\infty} \frac{d_{k, w}}{4^{k}} \sum_{l=0}^{k} \sum_{i=0}^{\infty}(-1)^{n+l} 4^{n}\left(\begin{array}{c}
\frac{2}{w} l \\
i
\end{array}\right)\left(\begin{array}{c}
\frac{i}{2} \\
j
\end{array}\right) .
$$

\section{Symmetric identities of Catalan-Daehee numbers}

Let $p$ be a fixed prime number. Throughout this paper, $\mathbb{Z}_{p}, \mathbb{Q}_{p}$ and $\mathbb{C}_{p}$ will denote the ring of $p$-adic integers, the field of $p$-adic rational numbers and the completion of algebraic closure of $\mathbb{Q}_{p}$. The $p$-adic norm $|\cdot|_{p}$ is normalized as $|p|_{p}=\frac{1}{p}$. Let $f(x)$ be a uniformly differential function $f: \mathbb{Z}_{p} \rightarrow \mathbb{C}_{p}$, the $p$-adic invariant integral $I_{1}(f)$ is given by

$$
\begin{aligned}
I_{1}(f) & =\int_{\mathbb{Z}_{p}} f(x) d \mu(x) \\
& =\lim _{N \rightarrow \infty} \frac{1}{p^{N}} \sum_{x=0}^{p^{N}-1} f(x),
\end{aligned}
$$

(see $[1,2,4,6,8,11,17,20]$.

By taking $f_{1}(x)=f(x+1)$, the following integral equation is well-known

$$
I_{1}\left(f_{1}\right)=I_{1}(f)+f^{\prime}(0)
$$

where $f^{\prime}(0)=\left.\frac{d f(x)}{d x}\right|_{x=0}$. 
Note that

$$
\begin{aligned}
\int_{\mathbb{Z}_{p}}(1-4 t)^{\frac{w(x+y)}{2}} d \mu(y) & =\frac{\frac{1}{2} \log (1-4 t)}{\sqrt{(1-4 t)^{w}}-1}(1-4 t)^{\frac{w x}{2}} \\
& =\sum_{n=0}^{\infty} d_{n, w}(x) t^{n}
\end{aligned}
$$

and since

$$
\begin{aligned}
\int_{\mathbb{Z}_{p}}(1-4 t)^{\frac{w(x+y)}{2}} d \mu(y) & =\int_{\mathbb{Z}_{p}} \sum_{n=0}^{\infty}\left(\begin{array}{c}
\frac{w(x+y)}{2} \\
n
\end{array}\right)(-4 t)^{n} d \mu(y) \\
& =\sum_{n=0}^{\infty} \int_{\mathbb{Z}_{p}}\left(\begin{array}{c}
\frac{w(x+y)}{2} \\
n
\end{array}\right) d \mu(y)(-4 t)^{n}
\end{aligned}
$$

we have

$$
\int_{\mathbb{Z}_{p}}\left(\begin{array}{c}
\frac{w(x+y)}{2} \\
n
\end{array}\right) d \mu(y)=\frac{(-1)^{n}}{4^{n}} d_{n, w}(x) .
$$

Let us observe that

$$
\begin{aligned}
\frac{2}{w \log (1-4 t)} & \left(\int_{\mathbb{Z}_{p}}(1-4 t)^{\frac{w(x+n)}{2}} d \mu(y)-\int_{\mathbb{Z}_{p}}(1-4 t)^{\frac{w x}{2}} d \mu(y)\right) \\
& =\sum_{i=0}^{n-1}(1-4 t)^{\frac{w i}{2}} \\
& =\sum_{i=0}^{n-1} \sum_{k=0}^{\infty}\left(\frac{w i}{2}\right)^{k} \frac{1}{k !}(\log (1-4 t))^{k} \\
& =\sum_{i=0}^{n-1} \sum_{k=0}^{\infty}\left(\frac{w i}{2}\right)^{k} \sum_{m=k}^{\infty} S_{1}(m, k)(-4)^{m} \frac{t^{m}}{m !} \\
& =\sum_{m=0}^{\infty}(-1)^{m} \sum_{k=0}^{m} w^{k} \sum_{i=0}^{n-1} i^{k} 2^{2 m-k} S_{1}(m, k) \frac{t^{m}}{m !} \\
& =\sum_{m=0}^{\infty}\left(\sum_{k=0}^{m} w^{k} \sum_{i=0}^{n-1} i^{k} 2^{2 m-k}\left|S_{1}(m, k)\right|\right) \frac{t^{m}}{m !},
\end{aligned}
$$

where $S_{1}(m, k)$ denote the Stirling numbers of the first kind, so $\left|S_{1}(m, k)\right|$ mean the unsigned Stirling numbers of the first kind.

For simplicity, from now on we use $S_{k}(n)$ to denote $\sum_{i=0}^{n-1} i^{k}$ and

$$
T_{m}(n, w)=\sum_{k=0}^{m} w^{k} S_{k}(n) 2^{2 m-k}\left|S_{1}(m, k)\right| .
$$

Then the equation (15) becomes

$$
\begin{aligned}
\frac{2}{w \log (1-4 t)} & \left(\int_{\mathbb{Z}_{p}}(1-4 t)^{\frac{w(x+y)}{2}} d \mu(y)-\int_{\mathbb{Z}_{p}}(1-4 t)^{\frac{w x)}{2}} d \mu(y)\right) \\
& =\sum_{m=0}^{\infty} T_{m}(n, w) \frac{t^{m}}{m !} .
\end{aligned}
$$


For $w_{1}, w_{2} \in \mathbb{N}$, we set

$$
I^{(m)}\left(w_{1} w_{2}\right)=\frac{\int_{\mathbb{Z}_{p}^{m}}(1-4 t)^{\frac{w_{1}\left(\sum_{m} \mathbf{x}+w_{2} x\right)}{2}} d \mathbf{x} \int_{\mathbb{Z}_{p}^{m}}(1-4 t)^{\frac{w_{2}\left(\sum_{m} \mathbf{x}+w_{1} x\right)}{2}} d \mathbf{x}}{\int_{\mathbb{Z}_{p}}(1-4 t)^{\frac{w_{1} w_{2}}{2} x} d x},
$$

where $\int_{\mathbb{Z}_{p}^{m}} f\left(x_{1}, x_{2}, \cdots, x_{m}\right) d \mathbf{x}=\int_{\mathbb{Z}_{p}} \cdots \int_{\mathbb{Z}_{p}} f\left(x_{1}, x_{2}, \cdots, x_{m}\right) d x_{1} d x_{2} \cdots d x_{m} \quad$ and $\sum_{m} \mathbf{x}=x_{1}+x_{2}+\cdots+x_{m}$.

Note that $I^{(m)}\left(w_{1}, w_{2}\right)$ is symmetric in $w_{1}$ and $w_{2}$.

From (17), we have

$$
\begin{aligned}
I^{(m)}\left(w_{1}, w_{2}\right) & =\left(\int_{\mathbb{Z}_{p}^{m}}(1-4 t)^{\frac{w_{1}\left(\sum_{m} \mathbf{x}\right)}{2}} d \mathbf{x}\right)(1-4 t)^{\frac{w_{1} w_{2} x}{2}} \\
& \times\left(\frac{\int_{\mathbb{Z}_{p}}(1-4 t)^{\frac{w_{2} x_{m}}{2}} d x_{m}}{\int_{\mathbb{Z}_{p}}(1-4 t)^{\frac{w_{1} w_{2}}{2} x} d x}\right) \\
& \times\left(\int_{\mathbb{Z}_{p}^{m-1}}(1-4 t)^{\frac{w_{2}\left(\sum_{m-1} \mathbf{x}\right)}{2}} d x_{1} d x_{2} \cdots d x_{m-1}\right)(1-4 t)^{\frac{w_{1} w_{2} y}{2}} .
\end{aligned}
$$

It is not difficult to show that

$$
\begin{aligned}
\frac{2}{w \log (1-4 t)} & \left(\int_{\mathbb{Z}_{p}}(1-4 t)^{\frac{w(x+n)}{2}} d x-\int_{\mathbb{Z}_{p}}(1-4 t)^{\frac{w x)}{2}} d x\right) \\
& =\frac{n \int_{\mathbb{Z}_{p}}(1-4 t)^{\frac{w x}{2}} d x}{\int_{\mathbb{Z}_{p}}(1-4 t)^{\frac{n w x}{2}} d x} \\
& =\sum_{k=0}^{\infty} T_{k}(w) \frac{t^{k}}{k !}
\end{aligned}
$$

and

$$
\begin{aligned}
(1-4 t)^{\frac{w_{1} w_{2}}{2} x} & \int_{\mathbb{Z}_{p}^{m}}(1-4 t)^{\frac{w_{1}\left(\sum_{m} \mathbf{x}\right)}{2}} d \mathbf{x} \\
& =\left(\frac{\frac{w_{1}}{2} \log (1-4 t)}{(1-4 t)^{\frac{w_{1}}{2}}-1}\right)^{m}(1-4 t)^{\frac{w_{1} w_{2}}{2} x} \\
& =\sum_{n=0}^{\infty} d_{n, w_{1}}^{(m)}\left(w_{2} x\right) t^{n} .
\end{aligned}
$$

Note that

$$
\begin{aligned}
\sum_{n=0}^{\infty} d_{n, w_{1}}^{(m)}\left(w_{2} x\right) t^{n} & =\left(\frac{\frac{w}{2} \log (1-4 t)}{(1-4 t)^{\frac{w x}{2}}-1}\right)^{m}(1-4 t)^{\frac{w x}{2}} \\
& =\left(\sum_{l=0}^{\infty} d_{l, w}^{(m)} t^{l}\right)\left(\sum_{k=0}^{\infty}\left(\begin{array}{c}
\frac{w x}{2} \\
k
\end{array}\right)(-4)^{k} t^{k}\right) \\
& =\sum_{n=0}^{\infty}\left(\sum_{k=0}^{n}\left(\begin{array}{c}
\frac{w x}{2} \\
k
\end{array}\right)(-1)^{k} 2^{2 k} d_{n-k, w}^{(m)}\right) t^{n}
\end{aligned}
$$


Hence

$$
d_{n, w}^{(m)}(x)=\sum_{k=0}^{n}\left(\begin{array}{c}
\frac{w x}{2} \\
k
\end{array}\right)(-1)^{k} 2^{2 k} d_{n-k, w}^{(m)} .
$$

From (17), we have

$$
\begin{aligned}
I^{(m)}\left(w_{1}, w_{2}\right) & =\left(\sum_{l=0}^{\infty} d_{l, w_{1}}^{(m)}\left(w_{2} x\right) t^{l}\right)\left(\sum_{k=0}^{\infty} T_{k}\left(w_{1} ; w_{2}\right) \frac{t^{k}}{k !}\right)\left(\sum_{i=0}^{\infty} d_{l, w_{1}}^{(m-1)}\left(w_{1} y\right) t^{i}\right) \frac{1}{w_{1}} \\
& =\frac{1}{w_{1}}\left(\sum_{l=0}^{\infty} d_{l, w_{1}}^{(m)}\left(w_{2} x\right) t^{l}\right)\left(\sum_{j=0}^{\infty}\left(\sum_{k=0}^{j} \frac{T_{k}\left(w_{1} ; w_{2}\right)}{k !} d_{j-k, w_{2}}^{(m-1)}(w, y)\right) t^{i}\right) \\
& =\sum_{l=0}^{\infty}\left(\frac{1}{w_{1}} \sum_{j=0}^{\infty} \sum_{k=0}^{j} \frac{T_{k}\left(w_{1} ; w_{2}\right)}{k !} d_{j-k, w_{2}}^{(m-1)}(w, y) d_{n-j, w_{1}}^{(m)}\left(w_{2} x\right)\right) t^{n}
\end{aligned}
$$

On the other hand, by the symmetric property of $I^{(m)}\left(w_{1}, w_{2}\right)$.

$$
\begin{aligned}
I^{(m)}( & \left.w_{2}, w_{1}\right)=\left(\int_{\mathbb{Z}_{p}^{m}}(1-4 t)^{\frac{w_{2}\left(\sum_{m} \mathbf{x}\right)}{2}} d \mathbf{x}\right)(1-4 t)^{\frac{w_{1} w_{2} x}{2}} \\
& \times\left(\frac{\int_{\mathbb{Z}_{p}}(1-4 t)^{\frac{w_{1} x_{m}}{2}} d x_{m}}{\int_{\mathbb{Z}_{p}}(1-4 t)^{\frac{w_{1} w_{2}}{2} x} d x}\right) \\
& \times\left(\int_{\mathbb{Z}_{p}^{m-1}}(1-4 t)^{\frac{w_{1}\left(\sum_{m-1} \mathbf{x}\right)}{2}} d x_{1} d x_{2} \cdots d x_{m-1}\right)(1-4 t)^{\frac{w_{1} w_{2} y}{2}} \\
= & \left(\sum_{l=0}^{\infty} d_{l, w_{2}}^{(m)}\left(w_{1} x\right) t^{l}\right)\left(\frac{1}{w_{2}} \sum_{k=0}^{\infty} T_{k}\left(w_{2} ; w_{1}\right) \frac{t^{k}}{k !}\right) \\
& \times\left(\sum_{i=0}^{\infty} d_{i, w_{2}}^{(m-1)}\left(w_{2} y\right) t^{i}\right) \\
= & \frac{1}{w_{2}}\left(\sum_{l=0}^{\infty} d_{l, w_{2}}^{(m)}\left(w_{1} x\right) t^{l}\right)\left(\sum_{j=0}^{\infty}\left(\sum_{k=0}^{j} \frac{T_{k}\left(w_{2} ; w_{1}\right)}{k !} d_{j-k, w_{2}}^{(m-1)}\left(w_{2} y\right)\right) t^{j}\right) \\
= & \sum_{n=0}^{\infty}\left(\frac{1}{w_{2}} \sum_{j=0}^{n} \sum_{k=0}^{j} \frac{T_{k}\left(w_{2} ; w_{1}\right)}{k !} d_{n-j, w_{2}}^{(m)}\left(w_{1} x\right) d_{j-k, w_{2}}^{(m-1)}\left(w_{2} y\right)\right) t^{n} .
\end{aligned}
$$

Therefore, by the symmetric property of $I^{(m)}\left(w_{1}, w_{2}\right)$ in $w_{1}$ and $w_{2}$, we obtain the following theorem.

Theorem 5. For $m, w_{1}, w_{2} \in \mathbb{N}, n \geq 0$, we have

$$
\begin{aligned}
& \frac{1}{w_{1}} \sum_{j=0}^{\infty} \sum_{k=0}^{j} \frac{T_{k}\left(w_{1} ; w_{2}\right)}{k !} d_{j-k, w_{2}}^{(m-1)}(w, y) d_{n-j, w_{1}}^{(m)}\left(w_{2} x\right) \\
& \quad=\frac{1}{w_{2}} \sum_{j=0}^{n} \sum_{k=0}^{j} \frac{T_{k}\left(w_{2} ; w_{1}\right)}{k !} d_{n-j, w_{2}}^{(m)}\left(w_{1} x\right) d_{j-k, w_{2}}^{(m-1)}\left(w_{2} y\right) .
\end{aligned}
$$


Now we observe that

$$
\begin{aligned}
I^{(m)}( & \left.w_{1}, w_{2}\right)=\left(\int_{\mathbb{Z}_{p}^{m}}(1-4 t)^{\frac{w_{1}\left(\sum_{m} \mathbf{x}\right)}{2}} d \mathbf{x}\right)(1-4 t)^{\frac{w_{1} w_{2} x}{2}} \\
& \times\left(\frac{\int_{\mathbb{Z}_{p}}(1-4 t)^{\frac{w_{2} x_{m}}{2}} d x_{m}}{\int_{\mathbb{Z}_{p}}(1-4 t)^{\frac{w_{1} w_{2}}{2} x} d x}\right) \\
& \times\left(\int_{\mathbb{Z}_{p}^{m-1}}(1-4 t)^{\frac{w_{2}\left(\sum_{m-1} \mathbf{x}\right)}{2}} d x_{1} d x_{2} \cdots d x_{m-1}\right)(1-4 t)^{\frac{w_{1} w_{2} y}{2}} \\
= & \frac{1}{w_{1}}\left(\int_{\mathbb{Z}_{p}^{m}}(1-4 t)^{\frac{w_{1}\left(\sum_{m} \mathbf{x}\right)}{2}} d \mathbf{x}\right)(1-4 t)^{\frac{w_{1} w_{2} x}{2}}\left(\sum_{i=0}^{w_{1}-1}(1-4 t)^{\frac{w_{2}}{2}}\right) \\
& \times\left(\int_{\mathbb{Z}_{p}^{m-1}}(1-4 t)^{\frac{w_{2}\left(\sum_{m-1} \mathbf{x}\right)}{2}} d x_{1} d x_{2} \cdots d x_{m-1}\right)(1-4 t)^{\frac{w_{1} w_{2} y}{2}} \\
= & \frac{1}{w_{1}} \sum_{i=0}^{w_{1}-1}\left(\int_{\mathbb{Z}_{p}^{m}}(1-4 t)^{\frac{w_{1}\left(\sum_{m} \mathbf{x}+\frac{w_{2}}{w_{1}} i+w_{2} x\right)}{2}} d \mathbf{x}\right) \\
& \times\left(\int_{\mathbb{Z}_{p}^{m-1}}(1-4 t)^{\frac{w_{2}\left(\sum_{m-1} \mathbf{x}\right)}{2}} d x_{1} d x_{2} \cdots d x_{m-1}\right) \\
= & \frac{1}{w_{1}}\left(\sum_{i=0}^{w_{1}-1} \sum_{k=0}^{\infty} d_{k, w_{1}}^{(m)}\left(w_{2} x+\frac{w_{2}}{w_{1}} i\right) t^{k}\right)\left(\sum_{l=0}^{\infty} d_{l, w_{2}}^{(m-1)}\left(w_{1} y\right) t^{l}\right) \\
= & \sum_{n=0}^{\infty}\left(\frac{1}{w_{1}} \sum_{k=0}^{n} \sum_{i=0}^{w_{1}-1} d_{k, w_{1}}^{(m)}\left(w_{2} x+\frac{w_{2}}{w_{1}} i\right) d_{n-k, w_{2}}^{(m-1)}\left(w_{1} y\right)\right) t^{n}
\end{aligned}
$$

On the other hand, we set

$$
\begin{aligned}
I^{(m)} & \left(w_{2}, w_{1}\right)=\left(\int_{\mathbb{Z}_{p}^{m}}(1-4 t)^{\frac{w_{2}}{2}\left(\sum_{m} \mathbf{x}\right)} d \mathbf{x}\right)(1-4 t)^{\frac{w_{1} w_{2}}{2} x}\left(\frac{\int_{\mathbb{Z}_{p}}(1-4 t)^{\frac{w_{1}}{2} x_{m}} d x_{m}}{\int_{\mathbb{Z}_{p}}(1-4 t)^{\frac{w_{1} w_{2}}{2} x} d x}\right) \\
& \times\left(\int_{\mathbb{Z}_{p}^{m-1}}(1-4 t)^{\frac{w_{1}}{2}\left(\sum_{m-1} \mathbf{x}\right)} d x_{1} d x_{2} \cdots d x_{m-1}\right)(1-4 t)^{\frac{w_{1} w_{2}}{2} y} \\
& =\frac{1}{w_{2}}\left(\int_{\mathbb{Z}_{p}^{m}}(1-4 t)^{\frac{w_{2}}{2}\left(\sum_{m} \mathbf{x}\right)} d \mathbf{x}\right)(1-4 t)^{\frac{w_{1} w_{2}}{2} x}\left(\sum_{i=0}^{w_{2}-1}(1-4 t)^{\frac{w_{1}}{2} i}\right) \\
& \times\left(\int_{\mathbb{Z}_{p}^{m-1}}(1-4 t)^{\frac{w_{1}}{2}\left(\sum_{m-1} \mathbf{x}+w_{2} y\right)} d x_{1} d x_{2} \cdots d x_{m-1}\right) \\
& =\frac{1}{w_{2}} \sum_{i=0}^{w_{2}-1}\left(\int_{\mathbb{Z}_{p}^{m}}(1-4 t)^{\frac{w_{2}}{2}\left(\sum_{m} \mathbf{x}+\frac{w_{1}}{w_{2}} i+w_{1} x\right)} d \mathbf{x}\right) \\
& \times\left(\int_{\mathbb{Z}_{p}^{m-1}}(1-4 t)^{\frac{w_{1}}{2}\left(\sum_{m-1} \mathbf{x}+w_{2} y\right)} d x_{1} d x_{2} \cdots d x_{m-1}\right)
\end{aligned}
$$




$$
\begin{aligned}
& =\frac{1}{w_{2}}\left(\sum_{i=0}^{w_{2}-1} \sum_{k=0}^{\infty} d_{k, w_{2}}^{(m)}\left(w_{1} x+\frac{w_{1}}{w_{2}} i\right) t^{k}\right)\left(\sum_{l=0}^{\infty} d_{l, w_{1}}^{(m-1)}\left(w_{2} y\right) t^{l}\right) \\
& =\sum_{n=0}^{\infty}\left(\frac{1}{w_{2}} \sum_{k=0}^{n} \sum_{i=0}^{w_{2}-1} d_{k, w_{2}}^{(m)}\left(w_{1} x+\frac{w_{1}}{w_{2}} i\right) d_{n-k, w_{1}}^{(m-1)}\left(w_{2} y\right)\right) t^{n} .
\end{aligned}
$$

Therefore, by the symmetric property of $I^{(m)}\left(w_{1}, w_{2}\right)$ in $w_{1}$ and $w_{2}$, we obtain the following theorem.

Theorem 6. For $w_{1}, w_{2}, m \in \mathbb{N}$ and $n \geq 0$, we have

$$
\begin{aligned}
\frac{1}{w_{1}} \sum_{k=0}^{n} & \sum_{i=0}^{w_{1}-1} d_{k, w_{1}}^{(m)}\left(w_{2} x+\frac{w_{2}}{w_{1}} i\right) d_{n-k, w_{2}}^{(m-1)}\left(w_{1} y\right) \\
& =\frac{1}{w_{2}} \sum_{k=0}^{n} \sum_{i=0}^{w_{2}-1} d_{k, w_{2}}^{(m)}\left(w_{1} x+\frac{w_{1}}{w_{2}} i\right) d_{n-k, w_{1}}^{(m-1)}\left(w_{2} y\right) .
\end{aligned}
$$

Remark. Let $y=0$ and $m=1$, then we note that

$$
\begin{aligned}
& \frac{1}{w_{1}} \sum_{i=0}^{w_{1}-1} d_{k, w_{1}}\left(w_{2} x+\frac{w_{2}}{w_{1}} i\right) \\
&=\frac{1}{w_{2}} \sum_{i=0}^{w_{2}-1} d_{k, w_{2}}\left(w_{1} x+\frac{w_{1}}{w_{2}} i\right)
\end{aligned}
$$

Taking $w_{2}=1$, we have

$$
d_{k}\left(w_{1} x\right)=\frac{1}{w_{1}} \sum_{i=0}^{w_{1}-1} d_{n, w-1}\left(x+\frac{1}{w_{1}} i\right) .
$$

\section{Results and discussion}

In this paper, we have defined the $w$-Catalan-Daehee polynomials and numbers,

$$
\frac{\frac{1}{2} \log (1-4 t)}{\sqrt{(1-4 t)^{w}}-1}(1-4 t)^{\frac{w}{2} x}=\sum_{n=0}^{\infty} d_{n, w}(x) t^{n} .
$$

These are closely related Catalan, Daehee and Catalan-Daehee numbers. In Proposition 2, we gave an explicit formula for the $w$-Catalan-Daehee polynomials. In Propositions 1 and 3, we give relations between the $w$-Catalan-Daehee and Daehee numbers. Proposition 4 expresses a relation between the $w$-Catalan-Daehee and Catalan numbers.

In Section 3, we gave explicit expression for symmetric identities of the $w$-Catalan-Daehee polynomials, which are derived from $p$-adic invariant integral on $\mathbb{Z}_{p}$. 


\section{Conclusion}

In this paper, we defined and investigated the symmetric property of the $w$-Catalan-Daehee polynomials. In addition, by using the $p$-adic integral on $\mathbb{Z}_{p}$, we explicitly showed that the $w$-CatalanDaehee polynomials have symmetric identities from the $p$-adic invariant integral on $\mathbb{Z}_{p}$.

\section{Acknowledgement}

This research was supported by Basic Science Research Program through the National Research Foundation of Korea (NRF) funded by the Ministry of Education (2018-01-019).

\section{References}

[1] Choi, S. (2018) Linear symmetry of the modified $q$-Euler polynomials. Adv. Stud. Contemp. Math. (Kyungshang), 28 (2), 201-206.

[2] Choi, S., Kim, T., Kwon, H.-I., \& Kwon, J. (2018) Quadratic symmetry of modified $q$-Euler polynomials, Adv. Difference Equ., Paper No. 38, 9 pages.

[3] Dolgy, D. V., Jang, G.-W., Kim, D. S., \& Kim, T. (2017) Explicit Expressions for CatalanDaehee numbers, Proc. Jangjeon Math. Soc., 20(1), 1-9.

[4] Duran, U., Acikgoz, M., \& Araci, S. (2015) Symmetric identities involving weighted $q$ Genocchi polynomials under $S_{4}$. Proc. Jangjeon Math. Soc., 18 (4), 455-465.

[5] El-Desouky, B. S., \& Mustafa, A. (2016) New results on higher-order Daehee and Bernoulli numbers and polynomials, Adv. Difference Equ., 2016, Paper No. 32, 21 pages.

[6] He, Y. (2013) Symmetric identities for Carlitz's $q$-Bernoulli numbers and polynomials. Adv. Difference Equ., 2013:246, 10 pages.

[7] Jang, G.-W., Kwon, J., \& Lee, J. G. (2017) Some identities of degenerate Daehee numbers arising from nonlinear differential equation. Adv. Difference Equ., 2017, Paper No. 206, 10 pages.

[8] Jang, L.-C. (2011) A family of Barnes-type multiple twisted q -Euler numbers and polynomials related to Fermionic $p$-adic invariant integrals on $Z_{p}$, J. Comput. Anal. Appl., 13 (2), 376-387.

[9] Khan, W. A., Nisar, K. S., Duran, U., Acikgoz, M., \& Araci, S. (2018) Multifarious implicit summation formulae of Hermite-based poly-Daehee polynomials, Appl. Math. Inf. Sci., 12 (2), 305-310.

[10] Kim, D. S., \& Kim, T. (2017) Triple symmetric identities for $w$-Catalan polynomials, $J$. Korean Math. Soc., 54 (4), 1243-1264. 
[11] Kim, D. S., Lee, N., Na, J., \& Park, K. H. (2012) Identities of symmetry for higher-order Euler polynomials in three variables (I). Adv. Stud. Contemp. Math. (Kyungshang), 22 (1), $51-74$.

[12] Kim, T. (2016) A note in Catalan numbers associated with $p$-adic integral in $\mathbb{Z}_{p}$, Proc. Jangjeon Math. Soc., 19 (3), 493-501.

[13] Kim, T., \& Kim, D. S. (2017) Differential equations associated with Catalan-Daehee numbers and their applications, Revista de la Real Academia de Ciencias Exactas, Fisicas y Naturales. Serie A. Matematicas 111 (4), 1071-1081.

[14] Kim, T., Kim, D. S., \& Seo, J.-J. (2016) Symmetric identities for an analogue of Catalan polynomials, Proc. Jangjeon Math. Soc., 19 (3), 515-521.

[15] Koshy, T. (2009) Catalan Numbers with Applications. Oxford University Press, Oxford.

[16] Kwon, J., Sohn, G., \& Park, J.-W. (2018) Symmetric identities for $(h, q)$-extensions of the generalized higher order modified $q$-Euler polynomials. J. Comput. Anal. Appl., 24 (8), 1431-1438.

[17] Lim, D., Kwon, J. (2016) A note on poly-Daehee numbers and polynomials. Proc. Jangjeon Math. Soc., 19 (2), 219-224.

[18] Liu, C., \& Wuyungaowa, W. (2018) Application of probabilistic method on Daehee sequences, Eur. J. Pure Appl. Math., 11 (1), 69-78.

[19] Moon, E.-J., Rim, S.-H., Jin, J.-H., \& Lee, S.-J. (2010) On the symmetric properties of higher-order twisted $q$-Euler numbers and polynomials, Adv. Difference Equ., 2010, Art. ID 765259,8 pages.

[20] Park, J.-W. (2016) On the $\lambda$-Daehee polynomials with $q$-parameter. J. Comput. Anal. Appl., $20(1), 11-20$.

[21] Pyo, S.-S., Kim, T., \& Rim, S.-H. (2017) Identities of the degenerate Daehee numbers with the Bernoulli numbers of the second kind arising from nonlinear differential equation, $J$. Nonlinear Sci. Appl., 10, 6219-6228.

[22] Pyo, S.-S., Kim, T., \& Rim, S.-H. Degenerate Daehee numbers of the third kind (submitted).

[23] Simsek, Y. (2017) Identities on the Changhee numbers and Apostol-type Daehee polynomials, Adv. Stud. Contemp. Math. (Kyungshang), 27 (2), 199-212.

[24] Simsek, Y., \& Yardimci, A. (2016) Applications on the Apostol-Daehee numbers and polynomials associated with special numbers, polynomials, and $p$-adic integrals. Adv. Difference Equ., 2016, Paper No. 308, 14 pages.

[25] Stanley, R. P. (2015) Catalan numbers. Cambridge University Press, New York. 